\title{
Utility of Cardiopulmonary Exercise Testing (CPET) in the Post-COVID-19 Context: Retrospective Analysis of a Single Centre Experience.
}

Edward Parkes ( $\nabla$ edward.parkes@uhcw.nhs.uk)

University Hospitals Coventry and Warwickshire NHS Trust

Joanna Shakespeare

University Hospitals Coventry and Warwickshire NHS Trust

Timothy Robbins

University Hospitals Coventry and Warwickshire NHS Trust and Warwick Medical School

loannis Kyrou

University Hospitals Coventry and Warwickshire NHS Trust

Harpal Randeva

University Hospitals Coventry and Warwickshire NHS Trust

Asad Ali

University Hospitals Coventry and Warwickshire NHS Trust

\section{Research Article}

Keywords: Cardiopulmonary exercise testing (CPET), post-COVID-19 context, single centre experience, retrospective analysis

Posted Date: May 25th, 2021

DOl: https://doi.org/10.21203/rs.3.rs-537361/v1

License: (c) (i) This work is licensed under a Creative Commons Attribution 4.0 International License. Read Full License 


\section{Abstract}

Cardiopulmonary exercise testing (CPET) allows objective assessment of a patient's global response to maximal incremental exercise. CPET has been proposed to have a role in investigating post-COVID syndrome. However, CPET is resource intensive, and essential for restoration of other clinical services (e.g. cancer surgery). The aim of this study was to explore utility of CPET in assessing functional status of COVID-19 survivors with persistent dyspnoea. Of the 600 patients reviewed in a post-COVID-19 assessment clinic between May 2020 and April 2021, 12 (male/female: 8/4; age: 4 \pm 15.2 years; BMl: $32.8 \pm 5.9 \mathrm{~kg} / \mathrm{m} 2$; non-smokers/ smokers: $8 / 4$ ) were referred for CPET due to persistent breathlessness out-keeping with disease severity. Of these patients, 10 patients demonstrated reduced peak VO2, whilst five had an exercise limitation attributed to physical deconditioning. Two patients had mainly a cardiac limitation to exercise, with a further three patients demonstrating breathing pattern disorder, pulmonary vascular disease and lung disease. The findings of this single-centre study suggest that intensive CPET testing may not add substantial additional clinical information to aid patient investigation/management in the context of post-COVID. Such resource intensive procedures may be better utilised in selected patients and in the restoration of NHS services following the COVID-19 pandemic.

\section{Introduction}

Studies have identified a correlation between lung damage secondary to COVID-19 and reduction in lung function parameters, in particular indices of gas transfer [1]. However, there appears to be a disconnect between gas transfer impairment and exercise limitation as measured using cardiopulmonary exercise testing (CPET), with the suggestion that exercise limitation is primarily related to physical deconditioning rather than physiological impairment [1]. CPET is a symptom limited maximal incremental exercise test that allows a global assessment of a patient's response to exercise and provides a comprehensive assessment of a patient's ventilation, ventilatory-perfusion, cardiovascular and metabolic responses to incremental work. The performance of CPET allows certain important indices to be measured during the patient's physiological response to exercise. These measurements include, but are not limited to, maximal oxygen uptake $\left(\mathrm{VO}_{2}\right)$, relationship between ventilation and carbon dioxide output $\left(\mathrm{VE} / \mathrm{VCO}_{2}\right)$ and arterial oxygen saturation $\left(\mathrm{SpO}_{2}\right)$ [2]. These can be used to assess for the presence and severity of perfusionventilation and/or gas exchange abnormalities. Growing data indicate that COVID-19 can result in longer term clinical consequences, including the development of pulmonary interstitial disease and cardiovascular disease [3, 4], which may form part of the post-COVID syndrome. Consequently, CPET has been proposed as a potentially useful tool in the development of clinical management/support plans and future decision making for those with post-COVID-19 syndrome [5].

Data from the SARS outbreak in 2003 demonstrated long-term reduction in functional status even in the absence of any significant respiratory and/or cardiovascular impairment [6]. Assessment of exercise capacity in the short-term post-COVID period demonstrates decreased functional status [6, 7], and evidence of exercise induced desaturation [8]. This exercise impairment remains $2-3$ months post- 
discharge in approximately half of these patients $[9,10]$, however there is currently limited data on whether this persists in the long term.

With the anticipated long term healthcare requirements of patients with post-COVID-19 syndrome and the burden this will place on an already over-stretched NHS, it is imperative that all patients are able to access appropriate clinical investigations and subsequent treatments at the right time. As such, the aim of this pilot study was to evaluate the functional capacity of COVID-19 survivors by CPET, and assess its clinical value in investigating symptoms of post-COVID-19 syndrome.

\section{Methods}

This was a retrospective, single centre study. A respiratory consultant led post-COVID-19 assessment clinic was set-up at University Hospital, Coventry and Warwickshire (UHCW) NHS Trust as part of a local follow-up pathway for patients who recovered from COVID-19. All patients had a COVID-19 diagnosis confirmed by a positive reverse transcriptase-polymerase chain reaction (RT-PCR) or based on antibody testing, and were admitted to hospital for treatment or referred from post-COVID-19 assessment from primary care. Patients were referred for CPET due to severe persistent symptoms of breathlessness, as assessed by a Consultant Respiratory Physician or Specialist Respiratory Physiotherapist.

Patients performed CPET in accordance to local protocol based on the Preoperative Exercise Testing and Training Society (POETTS) recommendations [11]. As part of the CPET, patients also performed spirometry in accordance with the Association of Respiratory Technology and Physiology (ARTP) guidelines [12]. All tests were performed by a suitability qualified Healthcare Scientist. CPET and spirometry data were interpreted by a senior Healthcare Scientist using an evidence based approach [13]. The main outcome variable was peak $\mathrm{VO}_{2}$ defined as the highest oxygen uptake $\left(\mathrm{VO}_{2}\right)$ attained on a rapid incremental test at end-exercise [11]. This is reported in ml.min.kg and as a percentage of the maximal predicted $\mathrm{VO}_{2}\left(\mathrm{VO}_{2} \mathrm{max}\right)$. An abnormal peak $\mathrm{VO}_{2}$ was defined as $<85 \%$ patient predicted value [9].

This study was approved by the COVID-19 Research Committee at University Hospital, Coventry and Warwickshire NHS Trust (GAFREC Ref: 10015).

\section{Statistical analysis}

Continuous data are presented as mean and standard deviation (SD), with categorical data presented as percentages and frequencies. All analyses were performed using SPSS (IBM SPSS, Version 26, New York, USA).

\section{Results}

Out of 600 patients reviewed in the post-COVID-19 assessment clinic between May 2020 and April 2021, 12 (2\%; male/female: $8 / 4)$ reported persistent breathlessness out-keeping with disease severity and were referred for CPET. In these patients referred for CPET, COVID-19 was confirmed by PCR and antibody 
testing in 10/12 (83\%) and 2/12 (17\%), respectively. Mean age was 45 years (SD \pm 15.2 ), with a mean body mass index (BMI) of $32.8 \mathrm{~kg} / \mathrm{m}^{2}(\mathrm{SD} \pm 5.9)$. Of these patients, $8 / 12(66.6 \%)$ were non-smokers, whilst $5 / 12$ (42\%) had no pre-existing medical conditions, and $5 / 12(42 \%)$ had a previous diagnosis of asthma. Overall, $9 / 12(75 \%)$ patients were admitted to hospital due to the severity of their symptoms and received treatment. Of these patients, 5/12 (56\%) were admitted to Respiratory Wards and 4/12 (44\%) were admitted to Critical Care, one of whom received invasive mechanical ventilation. Mean time from hospital discharge to performance of pulmonary function testing was 88.8 days (SD \pm 79.1 ), and 182 (SD $\pm 110.9)$ days for CPET.

All patients performed spirometry successfully and in accordance with ARTP quality standards [13]. Spirometry data is summarised in Table 1 . Overall, $3 / 12(25 \%)$ of these patients demonstrated restrictive spirometry with a FVC below the lower limit of normal, whilst 1/12 (8\%) had a flow volume loop consistent with an upper airway obstruction and this individual was the only subject to be treated with invasive ventilation. No individuals had evidence of airflow obstruction.

Table 1

Spirometry Data.

\begin{tabular}{|lll|}
\hline Spirometry Indices & Mean & Standard Deviation \\
\hline FEV $_{1}$ L & 3.05 & 0.64 \\
\hline FEV $_{1} \%$ predicted & 86.12 & 13.71 \\
\hline FVC L & 3.77 & 0.80 \\
\hline FVC \% predicted & 85.5 & 12.49 \\
\hline FEV 1 /FVC \% & 81.24 & 7.03 \\
\hline PEF litres/second & 8.56 & 2.57 \\
\hline PEF \% predicted & 104.3 & 25.36 \\
\hline $\begin{array}{l}\text { FEV1 } \\
\text { flow Forced expiratory volume in one second; FVC = Forced vital capacity; PEF = Peak expiratory }\end{array}$ \\
\hline
\end{tabular}

CPET data is summarised in Table 2. Overall, $5 / 12(42 \%)$ of patients performed a maximal symptom limited CPET using $\geq 85 \%$ predicted HRmax as a cut-off value. Out of those patients who performed a sub-maximal CPET, bilateral leg fatigue was the predominant symptom that resulted in test termination. In total, $10 / 12(83 \%)$ of patients had a reduced peak $\mathrm{VO}_{2}$ percent predicted value. Of these 10 patients with a reduced peak $\mathrm{VO}_{2}, 5 / 10(50 \%)$ had an exercise limitation attributed to physical deconditioning. Two patients had mainly cardiac limitation to exercise, with a further three patients demonstrating breathing pattern disorder, pulmonary vascular disease and lung disease. No patients demonstrated ischaemic ECG changes. 
Table 2

Cardiopulmonary exercise test data.

\begin{tabular}{|c|c|c|}
\hline Cardiopulmonary Exercise Test Indices & Mean & Standard Deviation \\
\hline $\mathrm{VO}_{2} \mathrm{ml} / \mathrm{min} / \mathrm{kg}$ & 16.7 & 4.5 \\
\hline $\mathrm{VO}_{2} \%$ predicted & 65.1 & 13.7 \\
\hline Anaerobic threshold $\mathrm{ml} / \mathrm{min} / \mathrm{kg}$ & 11.0 & 2.8 \\
\hline Anaerobic threshold $\%$ predicted $\mathrm{VO}_{2} \max$ & 44.1 & 12.3 \\
\hline RER & 1.12 & 0.21 \\
\hline $\mathrm{O}_{2} / \mathrm{HR}(\mathrm{ml} /$ beat & 11.3 & 2.6 \\
\hline Eq. $\mathrm{CO}_{2}$ AT units & 33.7 & 6.6 \\
\hline $\mathrm{VO}_{2} / \mathrm{WR} \mathrm{ml} /$ watt & 8.87 & 1.9 \\
\hline Peak HR \% predicted & 80.2 & 12.6 \\
\hline $\mathrm{RR}$ & 35 & 11 \\
\hline BR \% predicted & 47.6 & 21 \\
\hline
\end{tabular}

\section{Discussion}

The COVID-19 pandemic in the UK has placed considerable strain on NHS diagnostic services [14]. Accordingly, there is a strong emphasis on appropriately utilising available diagnostic resources, such as CPET, ensuring that only those patients who are likely to gain benefit from specialised testing are referred. In the context of post-COVID patient follow-Up, CPET has been suggested as a potentially useful tool to guide clinical management plans; however, it constitutes a resource intensive procedure due to time, staffing, equipment and consumable costs.

The findings of the present study show a continued reduced functional status in the majority (83\%) of the studied post-COVID patients as defined by CPET at up to 12 months. This proportion appears to be higher than the reported in relation to SARS, where $41 \%$ of participants demonstrated a reduced peak $\mathrm{VO}_{2}$ at 3 months post infection [7]. There is also a reported reduction in peak $\mathrm{VO}_{2}$ of $34.5 \%$ and $55 \%$ in COVID-19 patients respectively within the same time frame $[9,10]$. The difference in this proportion may be attributed to the studied cohorts, since these studies were prospective in design and included a range of disease severity, while our patient cohort consisted of a selected group of post-COVID patients with persistent symptoms of breathlessness out-keeping with other radiological and physiological findings. 
Similarly, in a select group of 10 patients post COVID-19, all patients demonstrated a reduced peak $\mathrm{VO}_{2}$ [5].

Our study findings demonstrate that there is an initial and sustained impairment of functional status up to 12 months post COVID-19 in patients with persistent, ongoing breathlessness. This is predominately due to physical deconditioning which we postulate is related to muscle impairment and extended periods of inactivity. We have not identified a significant prevalence of any other clinical features within our study cohort, such as breathing pattern disorder or exercise induced desaturation. Where limitations other than physical deconditioning were identified, these conditions had previously been diagnosed and the performance of CPET served to confirm these causes of exercise limitation rather than identify them. Other studies have suggested that, although more simplistic and cheaper, field exercise tests including the $6 \mathrm{MST}$ and $6 \mathrm{MWT}$ are able to identify a reduced exercise capacity $[7,8]$; however, they lack the ability to accurately discriminate whether or not this is due to physical deconditioning or any other underlying pathology.

Our findings are consistent with the suggestion that functional impairment post COVID-19 is predominantly due to physical deconditioning based on previous research that reports a significant positive impact of pulmonary rehabilitation on exercise capacity in a group of elderly COVID-19 patients [15]. Considering these findings, we suggest that referral for diagnostic CPET should only occur if symptoms are exercise related. Nevertheless, before CPET can be utilised as a first line diagnostic test more evidence is required across all severity COVID-19 survivors, demonstrating that in the majority of patients physical deconditioning is the primary reason for symptoms and reduction in exercise capacity.

The appropriate triaging of patients to receive CPET is important given the need to manage extensive backlogs of operative patients [16] that have accumulated during the COVID-19 pandemic. Historically, CPET is primarily used for preoperative risk stratification and with a backlog of elective surgery, we argue that CPET should be prioritised for those patients who will gain the most benefit (e.g. those being assessed for major cancer surgery).

It is necessary to consider the limitations of our study, most notably the relatively small sample size and single centre nature of the study. However, despite this small sample size, we feel that this pilot study suggests that further research should initially focus on better understanding the physical deconditioning associated with the post-COVID-19 syndrome, rather than on CPET. Once we better understand both the physical deconditioning and recovery from this deconditioning, we should be better placed to identify atypical patients who may be presenting with an alternative problem, likely with a distinct underlying and potentially pre-existing pathology.

In conclusion, post-COVID-19 syndrome may present with profound symptoms and have a substantial negative impact on the quality of life for a large number of patients. It is therefore essential that these patients are supported through optimal diagnostic services and therapies, as clinically required. We believe that generalised CPET testing may not add significant additional clinical information that can be 
used to aid patient investigation and management in the context of post-COVID management, and that such investigations should be more focused to allow proper utilisation of NHS resources and restoration of routine NHS services.

\section{Declarations}

\section{Acknowledgements}

None

\section{Author contributions}

JS - Devised the topic of the manuscript, Drafted sections of the manuscript, Finalised the manuscript

TR - Drafted sections of the manuscript, Finalised the manuscript

IK - Finalised the manuscript

HR - Finalised the manuscript

AA - Finalised the manuscript

\section{Additional Information (including a Competing Interests Statement)}

None of the authors have any competing interests to declare.

\section{References}

1. Thomas M, Price OJ, Hull JH. Pulmonary function and COVID-19. Curr Opin Phys 2021;22.

2. Faghy MA, Sylvester KP, Cooper BG, Hull JH. Cardiopulmonary exercise testing in the COVID-19 endemic phase. Br J Anaesth. 2020;125(4):447-449.

3. George PM, Barratt SL, Condliffe R, et al. Respiratory follow-up of patients with COVID-19 pneumonia Thorax 2020;75:1009-1016.

4. Nishiga M, Wang DW, Han Y, et al. COVID-19 and cardiovascular disease: from basic mechanisms to clinical perspectives. Nat Rev Cardiol 2020;17:543-558.

5. Ong KC, Ng AWK, Lee LSU, et al. Pulmonary function and exercise capacity in survivors of severe acute respiratory syndrome $E R J 2004 ; 24(3): 436-442$

6. Daher A, Balfanz P, Cornelissen C, et al. Follow up of patients with severe coronavirus disease 2019 (COVID-19): Pulmonary and extrapulmonary disease sequelae. Respir Med 2020;174

7. Fuglebjerg NJU, Jensen TO, Hoyer N, et al. Silent hypoxia in patients with SARS CoV-2 infection before hospital discharge. Int J Infect Dis 2020;99:100-101 
8. Clavario P, de Marzo V, Lotti R, et al. Assessment of functional capacity with cardiopulmonary exercise testing in non-severe COVID-19 patients at three months follow-up. medRxiv 2020

9. Raman B, Cassar MP, Tunnicliffe EM, et al. Medium-term effects of SARS-CoV-2 infection on multiple vital organs, exercise capacity, cognition, quality of life and mental health, post-hospital discharge. EClinicalMedicine 2021;31:100683

10. Levett DZH, Jack S, Swart M, et al. Perioperative cardiopulmonary exercise testing (CPET): consensus clinical guidelines on indications, organization, conduct, and physiological interpretation. Br J Anaesth 2018;120(3):484 - 500

11. Sylvester KP, Clayton N, Cliff I, et al. ARTP statement on pulmonary function testing. BMJ Open Respir Res 2020;7:e000575

12. Wasserman KHJ, Sue D, Stringer W, Whipp BJ. Principles of exercise testing and interpretation. 4th ed. Philadelphia: Lippincott, Williams and Wilkins; 2004

13. Willan J, King AJ, Jeffery K, Bienz N. Challenges for NHS hospitals during covid-19 epidemic. BMJ 2020; 368:m1117

14. K Liu, W Zhang, Y Yang, J Zhang, Y Li, Y. Chen Respiratory rehabilitation in elderly patients with COVID-19: A randomized controlled study. Complement Ther Clin Pract 2020;39

15. Carr A, Smith J A, Camaradou J, Prieto-Alhambra D. Growing backlog of planned surgery due to covid-19. BMJ 2021; 372:n339 